\title{
Unidentified Line in X-Ray Spectra of the Andromeda Galaxy and Perseus Galaxy Cluster
}

\author{
A. Boyarsky, ${ }^{1}$ O. Ruchayskiy, ${ }^{2}$ D. Iakubovskyi, ${ }^{3,4}$ and J. Franse ${ }^{1,5}$ \\ ${ }^{1}$ Instituut-Lorentz for Theoretical Physics, Universiteit Leiden, Niels Bohrweg 2, 2333 CA Leiden, Netherlands \\ ${ }^{2}$ Ecole Polytechnique Fédérale de Lausanne, FSB/ITP/LPPC, BSP, CH-1015 Lausanne, Switzerland \\ ${ }^{3}$ Bogolyubov Institute of Theoretical Physics, Metrologichna Street 14-b, 03680 Kyiv, Ukraine \\ ${ }^{4}$ National University "Kyiv-Mohyla Academy", Skovorody Street 2, 04070 Kyiv, Ukraine \\ ${ }^{5}$ Leiden Observatory, Leiden University, Niels Bohrweg 2, 2333 CA Leiden, Netherlands \\ (Received 28 February 2014; revised manuscript received 14 October 2014; published 15 December 2014)
}

\begin{abstract}
We report a weak line at $3.52 \pm 0.02 \mathrm{keV}$ in $\mathrm{x}$-ray spectra of the Andromeda galaxy and the Perseus galaxy cluster observed by the metal-oxide-silicon (MOS) and $p$ - $n$ (PN) CCD cameras of the XMMNewton telescope. This line is not known as an atomic line in the spectra of galaxies or clusters. It becomes stronger towards the centers of the objects; is stronger for Perseus than for M31; is absent in the spectrum of a deep "blank sky" data set. Although for each object it is hard to exclude that the feature is due to an instrumental effect or an atomic line, it is consistent with the behavior of a dark matter decay line. Future (non-)detections of this line in multiple objects may help to reveal its nature.
\end{abstract}

DOI: 10.1103/PhysRevLett.113.251301

PACS numbers: $95.35 .+\mathrm{d}, 13.35 . \mathrm{Hb}, 14.60 . \mathrm{St}, 95.85 . \mathrm{Nv}$

The nature of dark matter (DM) is a question of crucial importance for both cosmology and for fundamental physics. As neutrinos - the only known particles that could be DM candidates - are too light to be consistent with various observations [1-4], it is widely anticipated that new particles should exist. Although many candidates have been put forward (see, e.g., Ref. [5]), little is known experimentally about the properties of DM particles: their masses, lifetimes, and interaction types remain largely unconstrained. A priori, a given DM candidate can possess a decay channel if its lifetime exceeds the age of the Universe. Therefore, the search for a DM decay signal provides an important test to constrain the properties of DM in a model-independent way. For fermionic particles, one should search above the Tremaine-Gunn limit [1] ( $\gtrsim 300 \mathrm{eV})$. If the mass is below $2 m_{e} c^{2}$, such a fermion can decay to neutrinos and photons with energy $E_{\gamma}=\frac{1}{2} m_{d m}$ [6]. One can search for such particles in $x$ rays [7,8] (see Ref. [9] for a review of previous searches). For each particular model, the particle's parameters are related by the requirement to provide the correct DM abundance. For example, for one very interesting DM candidate - the right-handed neutrino-this requirement restricts the mass range to $0.5-100 \mathrm{keV}[9,10]$. A large part of the available parameter space for sterile neutrinos is consistent with all astrophysical and cosmological bounds [11], and it is important to probe it further.

The DM decay line is much narrower than the spectral resolution of the existing $\mathrm{X}$-ray telescopes and, as previous searches have shown, should be rather weak. The x-ray spectra of astrophysical objects are crowded with weak atomic and instrumental lines, not all of which may be known. Therefore, even if the exposure of available observations continues to increase, it is hard to exclude an astrophysical or instrumental origin of any weak line found in the spectrum of an individual object. However, if the same feature is present in the spectra of many different objects, and its surface brightness and relative normalization between objects are consistent with the expected behavior of the DM signal, this can provide much more convincing evidence about its nature.

The present Letter takes a step in this direction. We present the results of the combined analysis of many XMM-Newton observations of two objects at different redshifts - the Perseus cluster $(z=0.0179$ [12] $)$ and the Andromeda galaxy (M31, $z=-0.001$ ), a Local Group member-together with a long exposure "blank sky" data set. (Each of the data sets used in previous decaying DM searches in M31 [13-17] had less statistics than we use. The nondetection of any signal in these works does not come in contradiction with our results.) We present the detection of a significant unmodeled excess at $3.52 \pm 0.02 \mathrm{keV}$ (rest frame) in both objects. We study its behavior and establish that it is consistent with a DM interpretation. However, as the line is weak $(\sim 4 \sigma$ in the combined data set) and the uncertainties in DM distribution are significant, positive detections or strong constraints from more objects are clearly needed to determine its nature.

Below we summarize the details of our data analysis and then discuss the results and caveats.

Data analysis.-We use the data obtained with the metaloxide-silicon (MOS) [18] and $p-n$ (PN) CCD [19] cameras of XMM-Newton ("XMM" in what follows). We use SAS v.13.0.0 [20] to reduce the raw data and filter the data for soft solar protons $[21,22]$ using the ESPFILT procedure. Because residual soft proton flares can produce weak linelike features in the spectra at positions where the effective area is non-monotonic (see, e.g., Ref. [23]), we apply the procedure described in Ref. [24], based on the comparison 

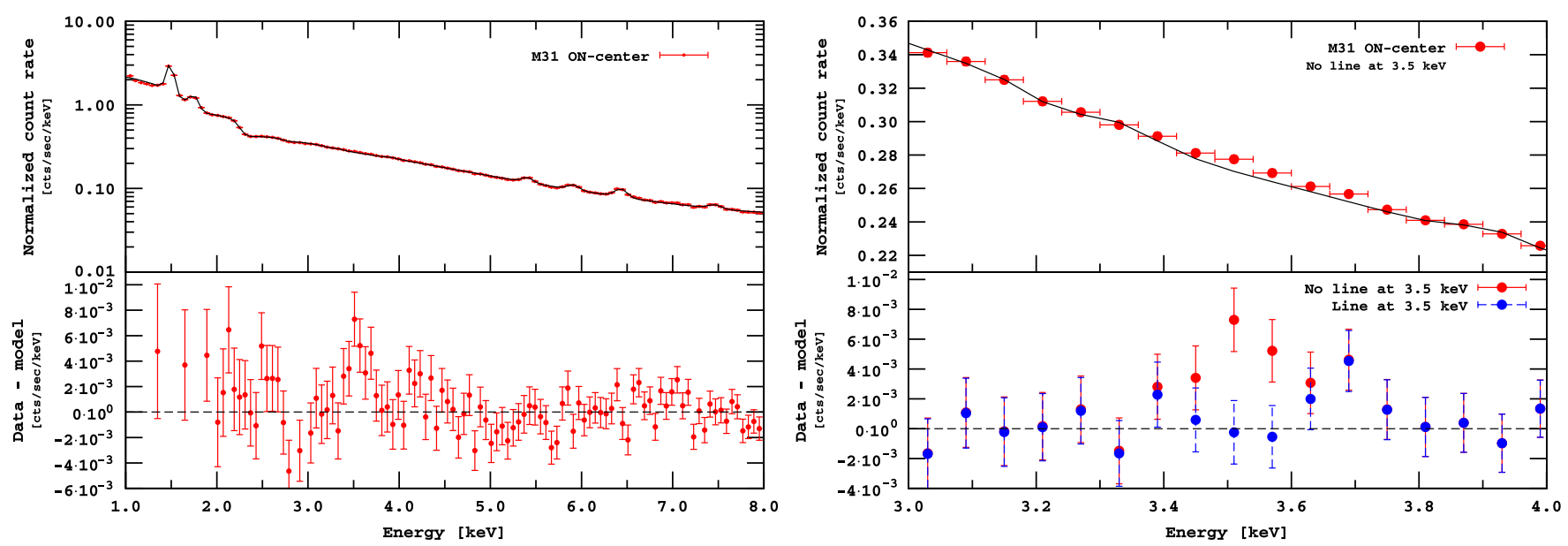

FIG. 1 (color online). Left: Folded count rate (top) and residuals (bottom) for the MOS spectrum of the central region of M31. Statistical $Y$ error bars on the top plot are smaller than the point size. The line around $3.5 \mathrm{keV}$ is not added, hence the group of positive residuals. Right: Zoom onto the line region.

of high-energy count rates for "in-field-of-view" (in-FOV) (10-15 arcmin off center) and out-FOV CCD regions [25]. We selected only observations where the ratio of $F_{\text {in }}-$ $F_{\text {out }}<1.15$. (Reference [24] argued that $F_{\text {in }}-F_{\text {out }}<1.3$ is a sufficient criterion for flare removal. We find by visual inspection of the resulting spectra that a stricter criterion is needed to reduce artificial linelike residuals [23,26]. Lowering the threshold further is not feasible as the statistical error bars on the value of $F_{\text {in }}-F_{\text {out }}$ are of the order of 5\%.)

Combined observation of M31.-We use $\sim 2 \mathrm{Msec}$ of raw exposure observations of M31 within the central $1.5^{\circ}$ (see Supplemental Material [27], Table II). We select from the XMM archive 29 MOS observations offset less than $1.5^{\prime}$ from the center of M31, and 20 MOS observations with offsets $23.7^{\prime}-55.8^{\prime}$ that passed our criterion for residual contamination. Not enough PN observations passed this test to include them. The central and off-center observations were co-added separately with the ADDSPEC routine from FTOOLS [31]. The resulting spectra were binned by $60 \mathrm{eV}$. This bin size is a factor $\sim 2$ smaller than the spectral resolution of the XMM at these energies, which makes the bins roughly statistically independent.

Background modeling. - We model the contribution of the instrumental (particle induced) background by a combination of an unfolded power law plus several narrow Gaussian lines. The positions and normalizations of the lines were allowed to vary freely and the most prominent instrumental $K \alpha$ lines $(\mathrm{Cr}, \mathrm{Mn}, \mathrm{K}, \mathrm{Fe}, \mathrm{Ni}, \mathrm{Ca}, \mathrm{Cu}$ ) and $\mathrm{Fe}$ $K \beta$ have been recovered. The width of the Gaussians was fixed at $1 \mathrm{eV}$ (an infinitely thin line for the XMM spectral resolution). We verified that allowing the line widths to vary freely leaves the results unchanged. We restrict our modeling to the energy interval $2-8 \mathrm{keV}$. The Galactic foreground is negligible above $2 \mathrm{keV}$ [32]. The combined emission of unresolved point sources at these energies is modeled by a POWERLAW [33]. Several linelike residuals around $2.4 \mathrm{keV}$ and $3.0 \mathrm{keV}$ were identified as $\mathrm{Ar}$ and $\mathrm{S}$ line complexes and the corresponding thin ( $1 \mathrm{eV}$ width) lines were added to the model. We verified that adding another POWERLAW component to model the contribution of the extragalactic x-ray background [24,32] does not improve the quality of fit and does not change the structure of the residuals.

Result.-The resulting spectrum of the central observations shows a group of positive residuals around $3.5 \mathrm{keV}$ (Fig. 1). Adding a thin Gaussian line at that energy reduces the total $\chi^{2}$ by $\sim 13$, see Table I (more than $3 \sigma$ significance for the extra 2 DOF). Examination of MOS1 and MOS2 observations individually finds the line in both cameras with comparable flux. For the off-center observations, none of the cameras show any detectable residual in the energy range $3.50-3.56 \mathrm{keV}$. The $2 \sigma$ upper bound on the flux is given in Table I.

Perseus cluster.-If the candidate weak signal is of astrophysical (rather than instrumental) origin, we should be able to detect its redshift. To this end we have chosen the nearby Perseus cluster (Abell 426). At its redshift the line's centroid would be shifted by $63 \mathrm{eV}$. As the position of the line is determined with about $30 \mathrm{eV}$ precision, one can expect to resolve the line's shift with about $2 \sigma$ significance.

We took 16 off-center observations of the Perseus cluster (see Supplemental Material [27], Table II) and processed them in the same way as for M31. The flare removal procedure left PN camera exposure, therefore, we also use PN data.

Background modeling.-The resulting spectra were then added together and fitted to the combination of VMEKAL (with free abundances for $\mathrm{Fe}, \mathrm{Ni}, \mathrm{Ar}, \mathrm{Ca}$ and $\mathrm{S}$ ) plus (extragalactic) POWERLAW. The instrumental background was modeled as in the M31 case.

Results.-The fit shows significant positive residuals at energies around $3.47 \mathrm{keV}$ (in the detector frame). Adding a 
TABLE I. Basic properties of combined observations used in this paper. Second column denotes the sum of exposures of individual observations. The improvement in $\Delta \chi^{2}$ when an extra line is added to a model is quoted for each data set. The last column shows the local significance of such an improvement when 2 extra DOF (position and flux of the line) are added. The energies for Perseus are quoted in the rest frame. Taking into account trial factors, the global (over three data sets) significance is $4.4 \sigma$ (see Supplemental Material [27] for details).

\begin{tabular}{lcccccc}
\hline \hline Data set & Exposure $(\mathrm{ksec})$ & $\chi^{2} /$ d.o.f. & Line position $(\mathrm{keV})$ & Flux $\left(10^{-6} \mathrm{counts} \mathrm{s}^{-1} \mathrm{~cm}^{-2}\right)$ & $\Delta \chi^{2}$ & Significance \\
\hline M31 on center & 978.9 & $97.8 / 74$ & $3.53 \pm 0.03$ & $4.9_{-1.3}^{+1.6}$ & 13.0 & $3.2 \sigma$ \\
M31 off center & 1472.8 & $107.8 / 75$ & $3.50-3.56$ & $<1.8(2 \sigma)$ & $\ldots$ & \\
Perseus cluster (MOS) & 628.5 & $72.7 / 68$ & $3.50 \pm 0.04$ & $7.0_{-2.6}^{+2.6}$ & 9.1 & $2.6 \sigma$ \\
Perseus cluster (PN) & 215.5 & $62.6 / 62$ & $3.46 \pm 0.04$ & $9.2_{-3.1}^{+3.1}$ & 8.0 & $2.4 \sigma$ \\
Perseus (MOS) & 1507.4 & $191.5 / 142$ & $3.52 \pm 0.02$ & $8.6_{-2.3}^{+2.2}$ (Perseus) & 25.9 & $4.4 \sigma$ \\
+M31 on center & & & & $4.6_{-1.4}^{+1.4}(\mathrm{M} 31)$ & $(3 \mathrm{DOF})$ \\
Blank sky & 15700.2 & $33.1 / 33$ & $3.45-3.58$ & $<0.7(2 \sigma)$ & $\ldots$ & \\
\hline \hline
\end{tabular}

ZGAUSS model with the redshift of the cluster improves the fit by $\Delta \chi^{2}=9.1$. The line's position is fully consistent with that of M31 (Table I). If we fix the position of the line to that of M31 and allow the redshift to vary, $z=0$ provides a worse fit by $\Delta \chi^{2}=3.6$ and its best-fit value is $(1.73 \pm 0.08) \times 10^{-2}$ - close to the value $z=0.0179$ which we have used.

Blank-sky data set.-To further study the origin of the new line and possible systematic effects we combine XMM blank-sky observations from Refs. [34,35] with observations of the Lockman Hole [36]. The data were reduced similarly to the other data sets. Figure 3 shows the combined spectrum. A data set with such a large exposure requires special analysis (as described in Ref. [26]). This analysis did not reveal any linelike residuals in the range $3.45-3.58 \mathrm{keV}$ with the $2 \sigma$ upper bound on the flux being $7 \times 10^{-7}$ counts $\mathrm{s}^{-1} \mathrm{~cm}^{-2}$. The closest detected linelike feature $\left(\Delta \chi^{2}=4.5\right)$ is at $3.67_{-0.05}^{+0.10} \mathrm{keV}$, consistent with the instrumental $\mathrm{Ca} K \alpha$ line. (Previously this line had only been observed in the PN camera [19].)

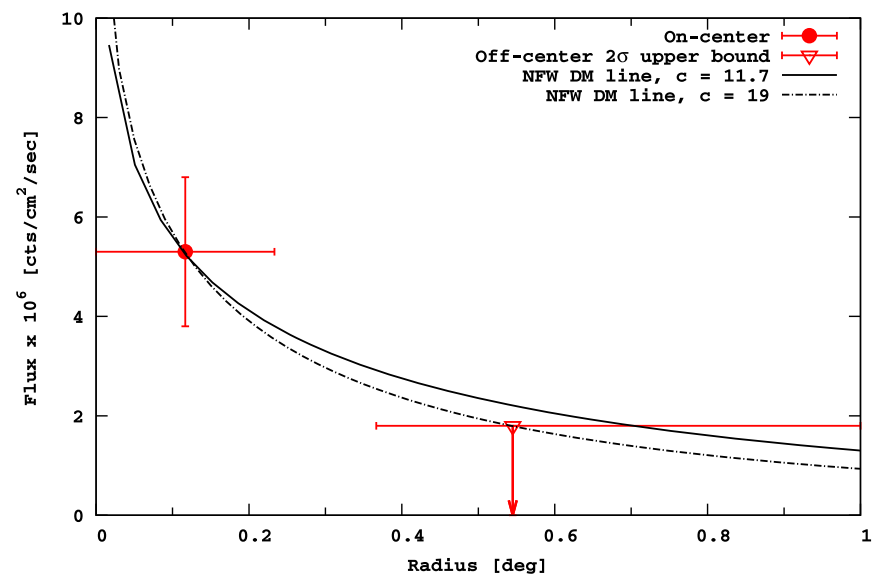

Combined fit of M31+ Perseus.-Finally, we have performed a simultaneous fit of the on-center M31 and Perseus data sets (MOS), keeping a common position of the line (in the rest frame) and allowing the line normalizations to be different. The line improves the fit by $\Delta \chi^{2}=25.9-4.4 \sigma$ significance (Table I).

Results and discussion. - We identified a spectral feature at $E=3.52 \pm 0.02 \mathrm{keV}$ in the combined data set of M31 and Perseus with a statistical significance $4.4 \sigma$ which does not coincide with any known line. Next we compare its properties with the expected behavior of a DM decay line.

The observed brightness of a decaying DM should be proportional to its column density $\mathcal{S}_{\mathrm{DM}}=\int \rho_{\mathrm{DM}} d \ell$-integral along the line of sight of the DM density distribution - and inversely proportional to the radiative decay lifetime $\tau_{\mathrm{DM}}$ :

$$
\begin{aligned}
F_{\mathrm{DM}} \approx & 2.0 \times 10^{-6} \frac{\text { counts }}{\mathrm{cm}^{2} \mathrm{sec}}\left(\frac{\Omega_{\mathrm{FOV}}}{500 \operatorname{arcmin}^{2}}\right) \\
& \times\left(\frac{\mathcal{S}_{\mathrm{DM}}}{500 \mathrm{M}_{\odot} / \mathrm{pc}^{2}}\right) \frac{10^{29} \mathrm{~s}}{\tau_{\mathrm{DM}}}\left(\frac{\mathrm{keV}}{m_{\mathrm{DM}}}\right) .
\end{aligned}
$$

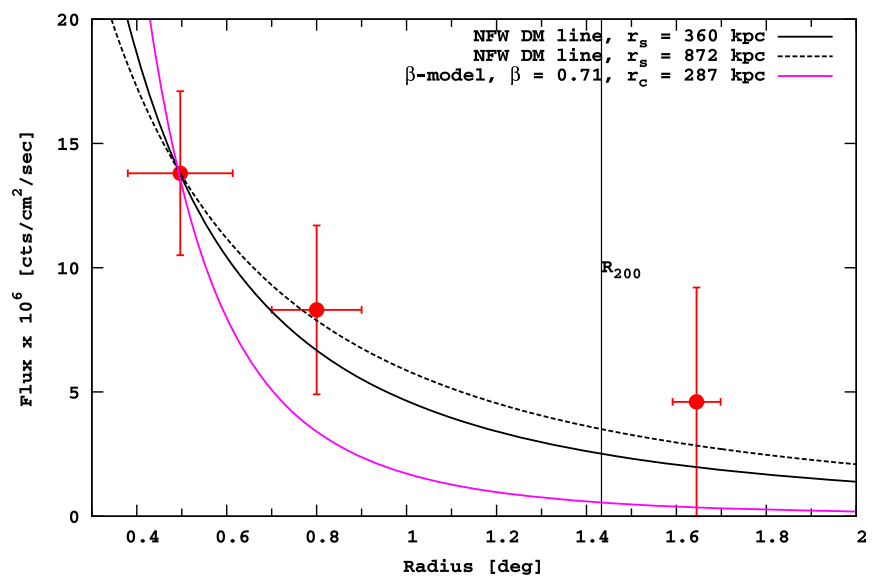

FIG. 2 (color online). The line's brightness profile in M31 (left) and the Perseus cluster (right). A NFW DM distribution is assumed, the scale $r_{s}$ is fixed to its best-fit values from Ref. [37] (M31) or Ref. [40] (Perseus) and the overall normalization is adjusted to pass through the left-most point. 


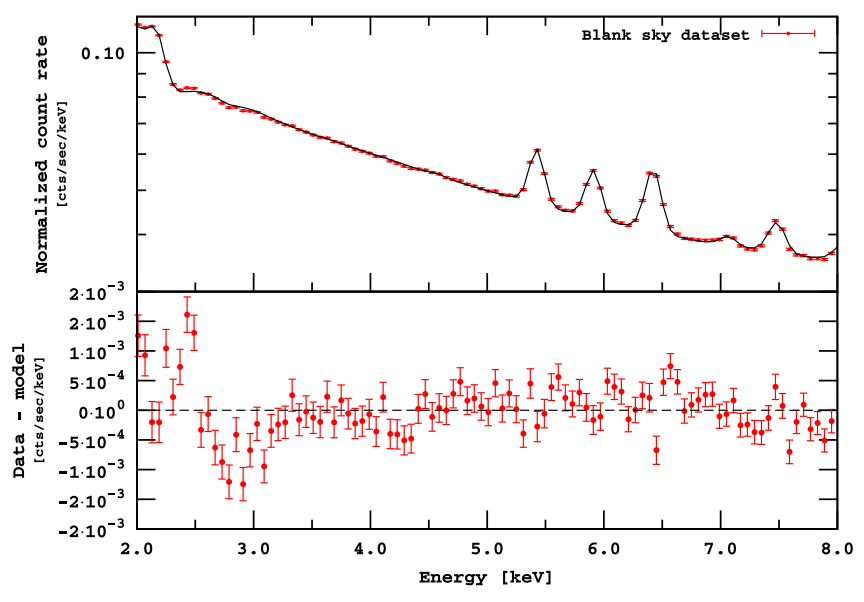

FIG. 3 (color online). Combination of 382 MOS blank sky observations.

M31 and Perseus brightness profiles.-Using the line flux of the center of M31 and the upper limit from the offcenter observations we constrain the spatial profile of the line. The DM distribution in M31 has been extensively studied (see an overview in Ref. [23]). We take NavarroFrenk-White (NFW) profiles for M31 with concentrations $c=11.7$ (solid line, [37]) and $c=19$ (dashed-dotted line). For each concentration we adjust the normalization so it passes through first data point (Fig. 2). The $c=19$ profile was chosen to intersect the upper limit, illustrating that the obtained line fluxes of M31 are fully consistent with the density profile of M31 (see, e.g., Refs. [37-39] for a $c=19-22$ model of M31).

For the Perseus cluster the observations can be grouped in three radial bins by their off-center angle. For each bin we fix the line position to its average value across Perseus $(3.47 \pm 0.07 \mathrm{keV})$. The obtained line fluxes together with $1 \sigma$ errors are shown in Fig. 2. For comparison, we draw the expected line distribution from DM decay using the NFW profile of Ref. [40] [best fit value $r_{s}=360 \mathrm{kpc}(c \approx 5)$, black solid line; upper bound $r_{s}=872 \mathrm{kpc}(c \approx 2)$, black dashed line]. The isothermal $\beta$ profile from Ref. [41] is shown in magenta. The surface brightness profile follows the expected DM decay line's distribution in Perseus.

Finally, we compare the predictions for the DM lifetime from the two objects. The estimated column density within the central part of M31 ranges between $\overline{\mathcal{S}} \sim 200-1000 \mathrm{M}_{\odot} / \mathrm{pc}^{2}$ with the average value being around $600 \mathrm{M}_{\odot} / \mathrm{pc}^{2}$ [23]. The column density of clusters follows from the $c-M$ relation [42-44]. Considering the uncertainty on the profile and that our observations of Perseus go beyond $r_{s}$, the column density in the region of interest is within $\overline{\mathcal{S}} \sim(100-600) \mathrm{M}_{\odot} / \mathrm{pc}^{2}$. Therefore, the ratio of expected signals between Perseus and the center of M31 can be $0.1-3.0$, consistent with the ratio of measured fluxes 0.7-2.7.

If DM is made of right-handed (sterile) neutrinos [45], the lifetime is related to its interaction strength (mixing angle):

$$
\begin{aligned}
\tau_{\mathrm{DM}} & =\frac{1024 \pi^{4}}{9 \alpha G_{F}^{2} \sin ^{2}(2 \theta) m_{\mathrm{DM}}^{5}} \\
& =7.2 \times 10^{29} \sec \left[\frac{10^{-8}}{\sin ^{2}(2 \theta)}\right]\left[\frac{1 \mathrm{keV}}{m_{\mathrm{DM}}}\right]^{5} .
\end{aligned}
$$

Using the data from M31 and taking into account uncertainties in its DM content, we obtain the mass $m_{\mathrm{DM}}=7.06 \pm 0.06 \mathrm{keV}$ and the mixing angle in the range $\sin ^{2}(2 \theta)=(2-20) \times 10^{-11}$ (taking the column density $\bar{S}=600 \mathrm{M}_{\odot} / \mathrm{pc}^{2}$ and using only statistical uncertainties on flux we would get $\sin ^{2}(2 \theta)=4.9_{-1.3}^{+1.6} \times 10^{-11}$ ). This value is fully consistent with previous bounds, Fig. 4. Moreover, it is intriguing that this value is consistent with the result of [46]. Indeed, our value of $\sin ^{2}(2 \theta)$ is based on completely independent analysis of the signal from M31 and our estimates for its DM content, whereas the result of Ref. [46] is based on the signal from stacked galaxy clusters and on the weighted DM column density from the full sample.

These values of $\sin ^{2}(2 \theta)$ mean that sterile neutrinos should be produced resonantly [48-50], which requires the presence of significant lepton asymmetry in primordial plasma at temperatures few hundreds $\mathrm{MeV}$. This produces restrictions on parameters of the $\nu$ MSM [10].

The position and flux of the discussed weak line are inevitably subject to systematical uncertainties. There are two weak instrumental lines ( $K \alpha \alpha$ at $3.31 \mathrm{keV}$ and $\mathrm{Ca} K \alpha$ at $3.69 \mathrm{keV}$ ), although formally their centroids are separated by more than $4 \sigma$. Additionally, the region below

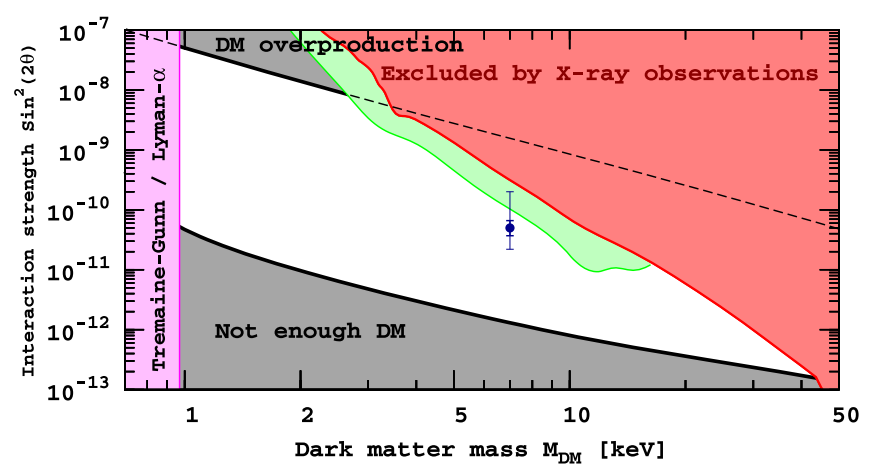

FIG. 4 (color online). Constraints on sterile neutrino DM within $\nu$ MSM [9]. Recent bounds from Refs. [16,17] are shown in green. Similar to older bounds (marked by red) they are smoothed and divided by factor 2 to account for possible DM uncertainties in M31. In every point in the white region sterile neutrinos constitute $100 \%$ of DM and their properties agree with the existing bounds. Within the gray regions too much (or not enough) DM would be produced in a minimal model like $\nu$ MSM. At masses below $\sim 1 \mathrm{keV}$ dwarf galaxies would not form $[4,47]$. The blue point would correspond to the best-fit value from M31 if the line comes from DM decay. Thick error bars are $\pm 1 \sigma$ limits on the flux. Thin error bars correspond to the uncertainty in the DM distribution in the center of M31. 
$3 \mathrm{keV}$ is difficult to model precisely, especially at large exposures, due to the presence of the absorption edge and galactic emission. However, although the residuals below $3 \mathrm{keV}$ are similar between the M31 data set (Fig. 1) and the blank sky data set (Fig. 3), the line is not detected in the latter.

If the feature were due to an unmodeled wiggle in the effective area, its flux would be proportional to the continuum brightness and the blank-sky data set would have exhibited a 4 times smaller feature with roughly the same significance (see Supplemental Material [27], Section B). In addition, the Perseus line would not be properly redshifted.

The properties of this line are consistent (within uncertainties) with the DM interpretation. To reach a conclusion about its nature, one will need to find more objects that give a detection or where nonobservation of the line will put tight constraints on its properties. The forthcoming Astro- $H$ mission [51] has sufficient spectral resolution to spectrally resolve the line against other nearby features and to detect the candidate line in the "strong line" regime [52]. In particular, Astro- $H$ should be able to resolve the Milky Way halo's DM decay signal and therefore all its observations can be used. Failure to detect such a line will rule out the DM origin of the Andromeda/Perseus signal presented here.

We thank D. Malyshev for collaboration; A. Neronov for useful critical comments; M. Shaposhnikov and M. Lovell for reading the manuscript and providing valuable comments. We also thank K. Abazajian, J. Beacom, and M. Kaplinghat for their comments. The work of D. I. was supported in part by the Swiss National Science Foundation Grant SCOPE IZ7370-152581, the Program of Cosmic Research of the National Academy of Sciences of Ukraine and the State Programme of Implementation of Grid Technology in Ukraine, and the grant of President of Ukraine for young scientists, and the grant of President of Ukraine for young scientists. The work of J. F. was supported by the De Sitter program at Leiden University with funds from Netherlands Organisation for Scientific Research. This research is part of the "Fundamentals of Science" program at Leiden University. This research has made use of the NASA/IPAC Extragalactic Database (NED), which is operated by the Jet Propulsion Laboratory, California Institute of Technology, under contract with the National Aeronautics and Space Administration.

Note added.-Recently Ref. [46] appeared, claiming a detection of a spectral feature at the same energy from a collection of galaxy clusters. Our analyses are independent, based on different data sets, but the results are fully consistent.

[1] S. Tremaine and J. E. Gunn, Phys. Rev. Lett. 42, 407 (1979).

[2] S. D. M. White, C. S. Frenk, and M. Davis, Astrophys. J. 274, L1 (1983).
[3] S. Hannestad and G. Raffelt, J. Cosmol. Astropart. Phys. 04 (2004) 008.

[4] A. Boyarsky, O. Ruchayskiy, and D. Iakubovskyi, J. Cosmol. Astropart. Phys. 03 (2009) 005.

[5] J. L. Feng, Annu. Rev. Astron. Astrophys. 48, 495 (2010).

[6] P. B. Pal and L. Wolfenstein, Phys. Rev. D 25, 766 (1982).

[7] K. Abazajian, G. M. Fuller, and W. H. Tucker, Astrophys. J. 562, 593 (2001).

[8] A. Dolgov and S. Hansen, Astropart. Phys. 16, 339 (2002).

[9] A. Boyarsky, D. Iakubovskyi, and O. Ruchayskiy, Phys. Dark Univ. 1, 136 (2012).

[10] A. Boyarsky, O. Ruchayskiy, and M. Shaposhnikov, Annu. Rev. Nucl. Part. Sci. 59, 191 (2009).

[11] A. Boyarsky, J. Lesgourgues, O. Ruchayskiy, and M. Viel, Phys. Rev. Lett. 102, 201304 (2009).

[12] The NASA/IPAC Extragalactic Database (NED), operated by the Jet Propulsion Laboratory, California Institute of Technology, under contract with the National Aeronautics and Space Administration.

[13] C. R. Watson, J. F. Beacom, H. Yuksel, and T. P. Walker, Phys. Rev. D 74, 033009 (2006).

[14] A. Boyarsky, D. Iakubovskyi, O. Ruchayskiy, and V. Savchenko, Mon. Not. R. Astron. Soc. 387, 1361 (2008).

[15] A. Boyarsky, O. Ruchayskiy, D. Iakubovskyi, M. G. Walker, S. Riemer-Sørensen, and S. H. Hansen, Mon. Not. R. Astron. Soc. 407, 1188 (2010).

[16] C. R. Watson, Z. Li, and N. K. Polley, J. Cosmol. Astropart. Phys. 3 (2012) 18.

[17] S. Horiuchi, P. J. Humphrey, J. Oñorbe, K. N. Abazajian, M. Kaplinghat, and S. Garrison-Kimmel, Phys. Rev. D 89, 025017 (2014).

[18] M. J. L. Turner et al., Astron. Astrophys. 365, L27 (2001).

[19] L. Strüder et al., Astron. Astrophys. 365, L18 (2001).

[20] Xmm-Newton Science Analysis System, http://xmm.esa int/sas/.

[21] A. M. Read and T. J. Ponman, Astron. Astrophys. 409, 395 (2003).

[22] K. D. Kuntz and S. L. Snowden, Astron. Astrophys. 478, 575 (2008).

[23] A. Boyarsky, O. Ruchayskiy, D. Iakubovskyi, M. G. Walker, S. Riemer-Sørensen, and S.H. Hansen, Mon. Not. R. Astron. Soc. 407, 1188 (2010).

[24] A. De Luca and S. Molendi, Astron. Astrophys. 419, 837 (2004).

[25] Fin_over_fout public script, v. 1.1, http://xmm.vilspa.esa.es/ external/xmm_sw_cal/background/Fin_over_Fout.

[26] D. Iakubovskyi, Ph.D. thesis, Leiden University, 2013.

[27] See Supplemental Material at http://link.aps.org/supplemental/ 10.1103/PhysRevLett.113.251301 for parameters of the XMM-Newton spectra of the Perseus cluster used in our analysis, that cites additional Refs. [28-30] as well as Ref. [18].

[28] P. Gondoin, B. Aschenbach, C. Erd, D. H. Lumb, S. Majerowicz, D. Neumann, and J. L. Sauvageot, in X-Ray and Gamma-Ray Instrumentation for Astronomy XI, Society of Photo-Optical Instrumentation Engineers (SPIE) Conference Series Vol. 4140, edited by K. A. Flanagan and O. H. Siegmund (SPIE, Bellingham, 2000), pp. 1-12.

[29] M. J. Turner et al., Astron. Astrophys. 365, L27 (2001).

[30] L. Struder et al., Astron. Astrophys. 365, L18 (2001).

[31] Irby, B., ftools webpage, HeaSoft, http://heasarc.gsfc.nasa .gov/docs/software/ftools/ftools_menu.html (2008). 
[32] J. Nevalainen, M. Markevitch, and D. Lumb, Astrophys. J. 629, 172 (2005).

[33] H. Takahashi, Y. Okada, M. Kokubun, and K. Makishima, Astrophys. J. 615, 242 (2004).

[34] J. Carter and A. Read, Astron. Astrophys. 464, 1155 (2007).

[35] D. B. Henley and R. L. Shelton, Astrophys. J. Suppl. Ser. 202, 14 (2012).

[36] H. Brunner, N. Cappelluti, G. Hasinger, X. Barcons, A. C. Fabian, V. Mainieri, and G. Szokoly, Astron. Astrophys. 479, 283 (2008).

[37] E. Corbelli, S. Lorenzoni, R. A. Walterbos, R. Braun, and D. A. Thilker, Astron. Astrophys. 511, A89 (2010).

[38] L. Chemin, C. Carignan, and T. Foster, Astrophys. J. 705, 1395 (2009).

[39] M. A. Sanchez-Conde, M. Cannoni, F. Zandanel, M. E. Gomez, and F. Prada, J. Cosmol. Astropart. Phys. 12 (2011) 011.

[40] A. Simionescu et al., Science 331, 1576 (2011).

[41] O. Urban et al., Mon. Not. R. Astron. Soc. 437, 3939 (2014).
[42] A. Boyarsky, A. Neronov, O. Ruchayskiy, and I. Tkachev, Phys. Rev. Lett. 104, 191301 (2010).

[43] L. King and J. Mead, Mon. Not. R. Astron. Soc. 416, 2539 (2011).

[44] R. Mandelbaum, U. Seljak, and C. M. Hirata, J. Cosmol. Astropart. Phys. 08 (2008) 006.

[45] S. Dodelson and L. M. Widrow, Phys. Rev. Lett. 72, 17 (1994).

[46] E. Bulbul, M. Markevitch, A. Foster, R. K. Smith, M. Loewenstein, and S. W. Randall, Astrophys. J. 789, 13 (2014).

[47] D. Gorbunov, A. Khmelnitsky, and V. Rubakov, J. Cosmol. Astropart. Phys. 10 (2008) 041.

[48] X.-D. Shi and G. M. Fuller, Phys. Rev. Lett. 82, 2832 (1999).

[49] M. Shaposhnikov, J. High Energy Phys. 08, (2008) 008.

[50] M. Laine and M. Shaposhnikov, J. Cosmol. Astropart. Phys. 6 (2008) 31.

[51] T. Takahashi et al., arXiv:1210.4378.

[52] A. Boyarsky, J. W. den Herder, A. Neronov, and O. Ruchayskiy, Astropart. Phys. 28, 303 (2007). 